\title{
PLC Networks with In-Band Full-Duplex Relays
}

\author{
Francisco J. Cañete*, Gautham Prasad ${ }^{\dagger}$, Lutz Lampe ${ }^{\dagger}$ \\ *Department of Ingeniería de Comunicaciones, ETSIT, Universidad de Málaga, Spain \\ ${ }^{\dagger}$ Department of Electrical and Computer Engineering, The University of British Columbia, Canada \\ Email: francis@ic.uma.es, \{gautham.prasad@alumni, lampe@ece\}.ubc.ca
}

\begin{abstract}
Electromagnetic compatibility regulations impose strict transmit power spectral density limitations on power line communication (PLC) that limits the coverage it can provide. As a consequence, PLC networks, especially those used in smart grid neighborhood area networks, employ one or more repeaters/relays for coverage enhancement. In this paper, we analyze the use of in-band full-duplex (IBFD) operation in relaying for power line networks. We present analyses and numerical results to demonstrate the superior operating performance of IBFD-based relay-aided networks over the legacy half-duplex ones, and contrast the extent of performance enhancement we achieve over using direct links. To estimate such performance, we focus on orthogonal frequency division multiplexing (OFDM) systems, which are standard in PLC. We investigate both decodeand-forward and amplify-and-forward schemes of relaying for dual-hop power line links, and quantify the performance in terms of the attainable data rate under diverse realistic power line channel and noise conditions. The fundamental analyses provided in this work guide the design of future IBFD relay-aided PLC networks across application scenarios.
\end{abstract}

\section{INTRODUCTION}

Power line communication (PLC) finds increasing applicability for networking in the smart grid due to its ability to transfer data over the existing infrastructure [1, Ch. 9], [2], [3]. PLC signals undergo high attenuation in these scenarios due to the large distances they traverse. As a result, multiple repeaters/relays are used in practical implementations to enhance coverage and guarantee connectivity to a broader range of users, especially when using the megahertz frequency band for PLC [4], [5]. Along the same lines, relaying is also applicable in large in-home environments when PLC may be used as a standalone infrastructure for multimedia communication or as a backbone network for $\mathrm{WiFi}$ range extension [6], and in a multi-dwelling environment where using PLC as a last-meter access technology may be appealing in lieu of delivering fiber to the apartments.

Two classical approaches for relaying are decode-andforward (DF) and amplify-and-forward (AF). DF schemes demand relatively complex relays that can decode the incoming information on an OFDM symbol-by-OFDM symbol basis and transmit the re-encoded data. While it enhances the network throughput, the overall performance may be limited by the overhead and delay generated by the relay nodes operating in a time division duplexed (TDD) manner. Alternative to DF, $\mathrm{AF}$ approaches allow the use of simpler relays, since they require no decoding, quantization, or digital signal processing

978-1-7281-4816-8/20/\$31.00 @2020 IEEE when working in the analog domain, by simply forwarding an amplified version of the incoming signal.

Relaying in PLC networks has been extensively studied in the past for both DF and AF schemes [7]-[10], with the assumption that PLC modems use TDD to accommodate upstream and downstream signals. The use of simultaneous bidirectional communication in the same frequency band, enabled by the in-band full-duplex (IBFD) operation [11][13], provides performance enhancement for both AF and DF methods of relaying by reducing the repeating delays associated at each relay node. Although integrating IBFD operation to enhance relaying performance has gathered attention in the domain of wireless communications [14]-[17], initial studies have only begun in the context of PLC [18]. We address this topic in the paper and provide the fundamental analyses that are essential for quantifying the performance of relay-aided PLC networks operating with IBFD relays.

The feasibility of IBFD for PLC was shown in [12] using a mixed domain approach to remove the transmitted signal self-interfering with the received signal-of-interest. The selfinterference (SI) is estimated digitally for computation efficiency and removed in the analog domain to counter the constraint of quantization noise at the receiver. In addition, a 4-to-2 wire hybrid converter is also used at the analog frontend of the PLC device to ensure impedance stability [11]. Such a solution is ideal when using IBFD with the DF scheme, whereas, for IBFD-AF, a simpler solution with only the hybrid circuit without any additional cancellation was shown to be sufficient in [18]. However, the design includes solving optimization problems to achieve frequency selective amplification and adapt impedances within the hybrid, which may be a challenge in practical implementation.

One of the other unique challenges we encounter in PLC in contrast to, say, wireless IBFD relay networks, is in designing the operation of the relay nodes. Regulatory bodies governing PLC do not impose a maximum PLC signal power limit, but rather set a restriction on the transmitted power spectral density (PSD) of PLC signals to restrict unintentional electromagnetic radiation. Therefore, a flat-amplification of the signal at an AF relay node may lead to the PSD mask being violated. Thus, a per-sub-carrier adaptive amplification is instead required. In this paper, we consider all the above aspects to analyze the performance of IBFD relaying networks in PLC.

Outline: The primary contribution of this work is to provide analyses of IBFD relay-aided PLC networks, for both DF 


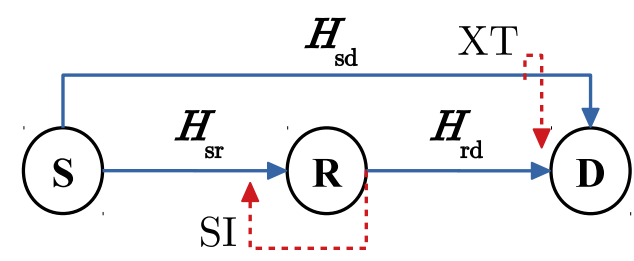

Fig. 1. The conceptual network that we consider for analysis.

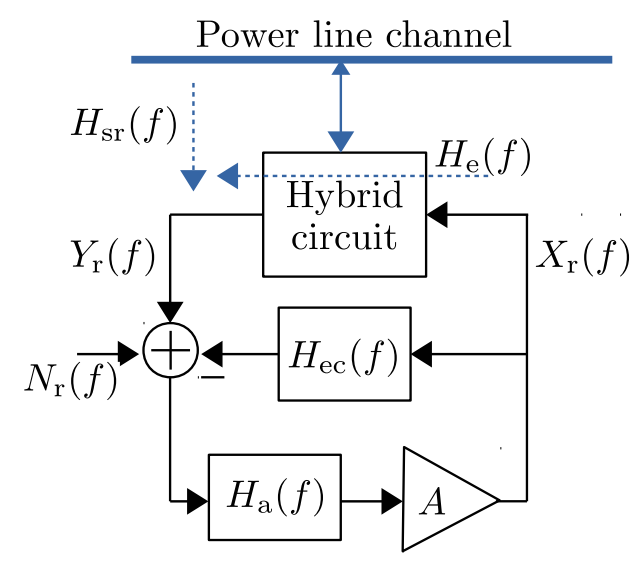

Fig. 2. A schematic block diagram of an IBFD relay node structure for AF relaying.

and $\mathrm{AF}$ schemes, under practical limitations including the adverse impact of residual SI resulting from different types of SI cancellation schemes, crosstalk, and quantization noise and distortion. We organize the paper as follows. We begin by describing the system model in Section II, followed by our analysis in Section III. In Section IV, we present our simulation results. Finally, we draw conclusions in Section V.

\section{System ModeL}

We consider an IBFD relay that can achieve simultaneous in-band data reception and forwarding, with the end-to-end signal flow being unidirectional. We conceptualize the network structure as shown in Fig. 1, where a source (S) transmits to the destination (D) via an optional relay node (R). The crosstalk (XT) caused by the direct link signal on the relayed one at D and the SI at R are highlighted in the figure for clarity. Since we consider unidirectional signal flow at any given time, we only require $\mathrm{R}$ to be IBFD enabled, and $\mathrm{S}$ and $\mathrm{D}$ may still function in the legacy half-duplex (HD) mode. We use $H_{\text {sr }}(f)$, $H_{\mathrm{rd}}(f), H_{\mathrm{sd}}(f)$, and $H_{\mathrm{e}}(f)$ to represent the channel response at any frequency $f$ of the links between $\mathrm{S} \rightarrow \mathrm{R}, \mathrm{R} \rightarrow \mathrm{D}, \mathrm{S} \rightarrow \mathrm{D}$, and $\mathrm{R}$ onto itself (echo or SI channel), respectively.

We also show a schematic block diagram of the relay node for the case of IBFD-AF relaying in Fig. 2, where $Y_{\mathrm{r}}(f)$, $N_{\mathrm{r}}(f)$, and $X_{\mathrm{r}}(f)$ are the input, received noise, and the output signals in the frequency domain, respectively. We use the hybrid design from [19] that exploits the reverse isolation provided by operational amplifiers to suppress the SI. We partition the amplification section of the relay node into two parts consisting of a flat gain with a power factor of $A^{2}$, and a frequency selective gain of $H_{\mathrm{a}}(f)$ that essentially attempts to compensate for $H_{\text {sr }}(f)$. By representing the response of the IBFD echo canceler as $H_{\mathrm{ec}}(f)$, we obtain the overall filter response of the relay node as

$$
H_{\mathrm{r}}(f)=\frac{A H_{\mathrm{a}}(f)}{1-A H_{\mathrm{a}}(f)\left[H_{\mathrm{e}}(f)-H_{\mathrm{ec}}(f)\right]} .
$$

We detail the strategies to choose $H_{\mathrm{a}}(f), A$, and $H_{\mathrm{ec}}(f)$ in Section III.

For the case of IBFD-DF relaying, we use a composite SI reduction technique with the hybrid suppression and an additional analog or digital cancellation from [11], [12] so that the signal from $\mathrm{S}$ can be effectively decoded and re-encoded before forwarding it to $\mathrm{D}$.

\section{Performance Analysis}

The objective of this section is to associate different strategies for the relay design with their corresponding achievable data rate expressions for the end-to-end network from $\mathrm{S}$ to $\mathrm{D}$.

\section{A. Formulation of Signal to Interference Plus Noise Ratio}

1) Individual Links: We compute the signal-to-noise ratio $(\mathrm{SNR})$ for the direct link $(\mathrm{S} \rightarrow \mathrm{D})$, the first hop $(\mathrm{S} \rightarrow \mathrm{R})$, and the second hop $(\mathrm{R} \rightarrow \mathrm{D})$ using

$$
\gamma_{i}(f)=\frac{S_{\mathrm{s}}(f)\left|H_{i}(f)\right|^{2}}{S_{\mathrm{Nr}}(f)+S_{\mathrm{E} i}},
$$

with $i=\mathrm{sd}, \mathrm{sr}, \mathrm{rd}$, respectively, and where $S_{\mathrm{s}}(f)$ represents the transmit PSD of the signal from the source, $S_{\mathrm{Nr}}(f)$ corresponds to that of the noise at the receiver and $S_{\mathrm{E} i}$ to that of the quantization error (flat) associated with the analogto-digital conversion. This latter value depends on the total power at the receiver input in each case, both of noise and signal components, as calculated in (16).

2) IBFD-AF: A primary drawback of using AF relaying is the noise amplification at the relay that increases the noise level at the destination. To address this issue, two solutions can be considered depending on the SI cancellation architecture implemented within the device. The feasibility of these solutions depend primarily on the hybrid circuit construction, e.g., how fast the hardware can process the receiving data to forward it. For an all-analog AF scheme that introduces no additional processing delays at the relays, the echo caused by simultaneous transmission and reception does not act as an interference, but is instead filtered by the feedback circuit at the relay (1) and can be tailored to ensure system stability [18]. Hence, the overall end-to-end channel only introduces linear distortion with noise enhancement. By further including the direct-link signal, whose effect under this setting does not act as crosstalk but instead as a linear multipath distortion, the equivalent overall channel response can be defined as ${ }^{1}$

$$
H_{\mathrm{T}}(f)=H_{\mathrm{sr}}(f) H_{\mathrm{r}}(f) H_{\mathrm{rd}}(f)+H_{\mathrm{sd}}(f) .
$$

\footnotetext{
${ }^{1}$ This condition requires that the transmission delay is negligible and the synchronization at D allows a coherent sum of both multipath signals (from $\mathrm{R}$ and $\mathrm{S}$ ).
} 
Under ideal cancellation of the echo and ideal frequency selective amplification at relay, i.e., perfect equalization of the SR channel response with $H_{\mathrm{r}}(f)=H_{\mathrm{sr}}^{-1}(f)$, (3) simplifies to

$$
H_{\mathrm{T}}(f)=H_{\mathrm{rd}}(f)+H_{\mathrm{sd}}(f) .
$$

Achieving $H_{\mathrm{r}}(f)=H_{\mathrm{sr}}^{-1}(f)$ also contributes to ensuring that the transmit PSD of the signal forwarded by the relay node complies with the PSD mask imposed by regulations. The resultant SNR at the destination is

$$
\begin{aligned}
\gamma_{\mathrm{d}, \mathrm{FD} 2-\mathrm{AF}}(f) & =\frac{S_{\mathrm{s}}(f)\left|H_{\mathrm{T}}(f)\right|^{2}}{K(f) \cdot S_{\mathrm{Nr}}(f)+S_{\mathrm{Nd}}(f)+S_{\mathrm{Ed}, \mathrm{FD} 2-\mathrm{AF}}} \\
& =\frac{S_{\mathrm{s}}(f)\left|H_{\mathrm{rd}}(f)+H_{\mathrm{sd}}(f)\right|^{2}}{(K(f)+1) S_{\mathrm{Nr}}(f)+S_{\mathrm{Ed}, \mathrm{FD} 2-\mathrm{AF}}},
\end{aligned}
$$

where $K(f)=\left|H_{\mathrm{r}}(f) H_{\mathrm{rd}}(f)\right|^{2}=\left|H_{\mathrm{sr}}^{-1}(f) H_{\mathrm{rd}}(f)\right|^{2}$, and for simplicity, we assume equal noise PSD at R and D, i.e., $S_{\mathrm{Nr}}(f)=S_{\mathrm{Nd}}(f)$. We refer to this AF scheme as FD2-AF.

However, when the relay incorporates digital processing or if the relay requires a non-trivial finite switching time between signal reception and transmission that is longer than the system sampling time, the input signal must be sampled and stored, and, hence, quantized, before being transmitted. In such a scenario, which we henceforth refer to as $F D 1-A F$, the echo can be considered to be uncorrelated with the desired signal and thus interferes at the relay when SI cancellation is not perfect. Therefore, a new signal to interference plus noise ratio (SINR) metric is required at relay, with an SI term at the denominator, leading to

$$
\gamma_{\mathrm{r}, \mathrm{FD} 1-\mathrm{AF}}(f)=\frac{S_{\mathrm{s}}(f)\left|H_{\mathrm{sr}}(f)\right|^{2}}{S_{\mathrm{Nr}}(f)+S_{\mathrm{r}}(f)\left|H_{\mathrm{re}}(f)\right|^{2}+S_{\mathrm{Er}, \mathrm{FD} 1-\mathrm{AF}}},
$$

where $S_{\mathrm{r}}(f)$ is the PSD of the signal transmitted by the relay, which can be the same as $S_{\mathrm{s}}(f)$ and be set based on the PLC mask mandated by regulations, and $H_{\mathrm{re}}(f)$ is the residual echo channel response. Under ideal conditions with perfect echo cancellation (or with nearly ideal SI removal), $\left|H_{\mathrm{re}}(f)\right|^{2} \rightarrow 0$ and (6) in turn equals (2). In such a case, and with perfect channel equalization at $\mathrm{R}$, the SINR at $\mathrm{D}$ for the overall channel is given by

$$
\begin{aligned}
\gamma_{\mathrm{d}, \mathrm{FD} 1-\mathrm{AF}}(f) & =\frac{S_{\mathrm{s}}(f)\left|H_{\mathrm{T}}(f)\right|^{2}}{(K(f)+1)\left(S_{\mathrm{Nr}}(f)+S_{\mathrm{Ed}, \mathrm{FD} 1-\mathrm{AF}}\right)+S_{\mathrm{XT}}(f)} \\
& =\frac{S_{\mathrm{s}}(f)\left|H_{\mathrm{rd}}(f)\right|^{2}}{(K(f)+1)\left(S_{\mathrm{Nr}}(f)+S_{\mathrm{Ed}, \mathrm{FD} 1-\mathrm{AF}}\right)+S_{\mathrm{XT}}(f)},
\end{aligned}
$$

where $S_{\mathrm{XT}}(f)$ represents the PSD of the crosstalk due to the direct-link signal, which is uncorrelated with the relayed one due to the processing delay. Hence, the overall channel response in (3) is $H_{\mathrm{T}}(f)=H_{\mathrm{sr}}(f) H_{\mathrm{r}}(f) H_{\mathrm{rd}}(f)=H_{\mathrm{rd}}(f)$, and

$$
S_{\mathrm{XT}}(f)=S_{\mathrm{s}}(f)\left|H_{\mathrm{sd}}(f)\right|^{2} \text {. }
$$

3) IBFD-DF: For the IBFD-DF case, we use the SI cancellation design from [12] for enabling IBFD operation, which cancels the echo in the analog domain but estimates the echo channel digitally in the frequency domain on an OFDM symbol-by-symbol basis. As a result, the echo is uncorrelated with the desired signal and the SINR at the relay node is

$$
\gamma_{\mathrm{r}, \mathrm{FD} j-\mathrm{DF}}(f)=\frac{S_{\mathrm{s}}(f)\left|H_{\mathrm{sr}}(f)\right|^{2}}{S_{\mathrm{Nr}}(f)+\mathrm{SI}_{\mathrm{r} j}(f)},
$$

where $\operatorname{SI}_{\mathrm{r} j}(f)$ is the PSD of the residual echo after SI cancellation. The value of $\mathrm{SI}_{\mathrm{r} j}(f)$ varies according to the SI cancellation scheme used to implement IBFD for three considered options, hence $j \in\{1,2,3\}$. The design in [12] recommends a run-time estimation of the SI in the presence of the signal-of-interest (SOI), i.e., estimating the echo without any silent periods. As a consequence, $\mathrm{SI}_{\mathrm{r} j}(f)$ depends on the relative signal powers of the SI and SOI, and on the noise and quantization error as well. In contrast, when the echo is estimated during a silent period in the absence of the SOI, the estimation accuracy, and therefore the value of $\mathrm{SI}_{\mathrm{r} j}(f)$ would be independent of the SOI. The design choice of SI estimation is application dependent, and we investigate the network performance with both forms of SI estimation at the relay node. Together with these, we also consider an IBFD solution from [11] that suggests echo estimation and cancellation to be performed entirely in the digital domain to achieve a costefficient implementation, albeit with a degraded cancellation performance. To this end, we consider the three types of SI cancellation designs: (i) mixed domain IBFD with run-time digital SI estimation and analog cancellation performed in the presence of the SOI, which we name as $F D 1-D F$, (ii) a fully digital cancellation scheme with digital SI estimation and removal, with the SI estimated during silent periods, which we refer to as $F D 2-D F$, and (iii) mixed domain IBFD with analog SI cancellation and digital echo estimation performed during silent periods, which we call FD3-DF. Hence, we obtain three different SINR $\gamma_{\mathrm{r}, \mathrm{FD} j \text {-DF }}(f)$ for $j \in\{1,2,3\}$.

Due to the signal regeneration in a DF scheme there is no noise amplification at the destination. Hence, the computation of $\gamma_{\mathrm{d}}(f)$ for the second hop corresponds to an independent link but with the crosstalk component. Signals from the direct and the relay-aided paths are uncorrelated because of the processing delay at the relay. Hence, a non-coherent interference appears with an additive term in SINR denominator, leading to

$$
\gamma_{\mathrm{d}, \mathrm{FD}-\mathrm{DF}}(f)=\frac{S_{\mathrm{r}}(f)\left|H_{\mathrm{rd}}(f)\right|^{2}}{S_{\mathrm{Nr}}(f)+S_{\mathrm{Ed}, \mathrm{FD}-\mathrm{DF}}+S_{\mathrm{XT}}(f)} .
$$

4) $H D-D F$ : In this $\mathrm{HD}$ scheme, $\mathrm{DF}$ guarantees that both hops are independent links like in the IBFD-DF case. At the second hop, there is no crosstalk from direct-link signal since sender is silent during relay transmission in the second timeduplexed slot. Hence, at the first hop, SINR can be calculated by using (2) with $i=\mathrm{sr}$, and at the second hop, by using it with $i=\mathrm{rd}$.

5) $H D-A F$ : In $\mathrm{HD}-\mathrm{AF}$ scheme, the signal quality at destination depends on the relay design. Under ideal conditions at the relay, the scenario is similar to that for FD1-AF but without 
crosstalk, because the sender is silent when relay transmits and the SINR at the destination can be calculated as

$$
\gamma_{\mathrm{d}, \mathrm{HD}-\mathrm{AF}}(f)=\frac{S_{\mathrm{s}}(f)\left|H_{\mathrm{rd}}(f)\right|^{2}}{(K(f)+1)\left(S_{\mathrm{Nr}}(f)+S_{\mathrm{Ed}, \mathrm{HD}-\mathrm{AF}}\right)} .
$$

\section{B. Impact of Quantization}

Throughout our analysis, we include the distortion introduced by quantization at the receiver during analog-to-digital signal conversion. To this end, we use the analysis in [12, Appendix A] to characterize the quantization error introduced by an analog-to-digital converter (ADC) that is tuned to operate on OFDM signals, such as those used in typical broadband PLC (BB-PLC) systems [20]. The signal-to-quantization noise and distortion (SINAD) is

$$
\gamma_{\mathrm{E}}=\frac{P_{\mathrm{inp}}}{P_{\mathrm{E}}}
$$

where $P_{\text {inp }}$ is the total power of the signal input into the quantizer and $P_{\mathrm{E}}$ is the quantization error power, which can be approximated for OFDM signals as [12, Appendix A]

$$
\gamma_{\mathrm{E}} \simeq 5.5 m-3.6,
$$

with $m$ being the nominal number of bits of the ADC. We use a 12-bit resolution for the ADC, that provides a reasonable SINAD for BB-PLC. The two power terms in (12) for a OFDM signal are

$$
\begin{gathered}
P_{\mathrm{E}}=S_{\mathrm{E}} N \Delta f, \\
P_{\text {inp }}=\sum_{k=1}^{N}\left(S_{\mathrm{r}}(k)+S_{\mathrm{n}}(k)\right) \Delta f,
\end{gathered}
$$

where $S_{\mathrm{r}}(k)$ and $S_{\mathrm{n}}(k)$ denote the PSD of incoming signal and noise respectively, $k$ is the OFDM sub-carrier index, $N$ the number of sub-carriers, and $\Delta f$ the sub-carrier frequency separation. Hence, the flat PSD of the quantization error is given by

$$
S_{\mathrm{E}}=\frac{\sum_{k=1}^{N}\left(S_{\mathrm{r}}(k)+S_{\mathrm{n}}(k)\right)}{(5.5 m-3.6) N} .
$$

\section{Achievable Rate Computation}

We consider a typical OFDM system with the transmitter adapting its modulation scheme on each sub-carrier using the channel state information (CSI) that it may obtain from the receiver as feedback. We use quadrature amplitude modulation (QAM) with a constellation size of $M$, and choose $M(k)$ such that

$$
\gamma(k)=(M(k)-1) \Gamma\left(P_{\mathrm{b}}\right),
$$

where $\gamma(k)$ is the SNR at the receiver, $\Gamma\left(P_{\mathrm{b}}\right)=\frac{-\ln 5 P_{\mathrm{b}}}{1.6}$ is the SNR gap of QAM w.r.t. the Gaussian channel capacity for a bit error rate of $P_{\mathrm{b}}$. Therefore, for any given SNR of $\gamma_{\mathrm{d}}$, we compute the data rate as

$$
C=\mathcal{B}\left[\gamma_{\mathrm{d}}\right]=\sum_{k=1}^{N} \log _{2}(M(k)) .
$$

The assumption of rate-adaptive links for IBFD relay-aided network may not always hold true, e.g., when the second hop has a lower link rate compared to the first. Therefore, we consider a fixed-rate link with the lower of the two link rates. However, for HD-DF relaying, we assume rate-adaptive links. In the following, we compute the data rates for each of the considered relaying strategies.

1) $H D-D F$ : The capacity for $\mathrm{HD}-\mathrm{DF}$ can be rate-adaptive in two cascaded independent links. Thus, the overall data rate is [9]

$$
C_{T}=\left[\frac{1}{C_{1}}+\frac{1}{C_{2}}\right]^{-1}
$$

with $C_{1}$ and $C_{2}$ being the data rate of the first and second hop, respectively, computed using (18).

2) $H D-A F$ : The capacity for an HD scheme with AF can be calculated under fixed-rate end-to-end conditions as

$$
C_{T}=\frac{1}{2} \mathcal{B}\left[\gamma_{\mathrm{d}}(f)\right]
$$

where the factor of $\frac{1}{2}$ is included to compensate for the two transmission instances required to complete the end-to-end communication, and $\gamma_{\mathrm{d}}(f)$ corresponds to the end-to-end link SNR given by (11).

3) IBFD-DF: The capacity for IBFD-DF corresponds to one of the two independent links at a fixed-rate, i.e.,

$$
C_{T}=\min \left[C_{1}, C_{2}\right],
$$

a result that applies for all the three considered configurations of FD1-DF, FD2-DF, and FD3-DF.

4) IBFD-AF: Finally, the capacity for IBFD-AF is analogous to the HD-AF condition but with only one transmission instance required to complete the end-to-end link, and therefore,

$$
C_{T}=\mathcal{B}\left[\gamma_{\mathrm{d}}(k)\right],
$$

where the function $\gamma_{\mathrm{d}}(k)$ is calculated by (7) or (5) for each case under study.

\section{NUMERICAL RESUlTS}

In this section, we provide numerical results of the data rates obtained for the different types of relaying strategies described in Section III using realistic in-home PLC channel and noise conditions.

\section{A. Simulation Settings}

1) Channel Generation: We consider a network topology shown in Fig. 3 and obtain the channel responses for this setup using the generator tool of [21]. The source and destination nodes are denoted as Tx and Rx, respectively, and are situated at two extreme ends of the network with the relay node, as indicated, in the central position between the two devices. We use indoor power cables with two parallel conductors with $2.5 \mathrm{~mm}^{2}$ cross sectional area ${ }^{2}$.

\footnotetext{
${ }^{2}$ Please refer to [21] for all other transmission line parameters and the methodology for the channel response computation. The only difference is that here, because link lengths are longer, a lower overestimation factor $\ell=2.5$ is selected.
} 


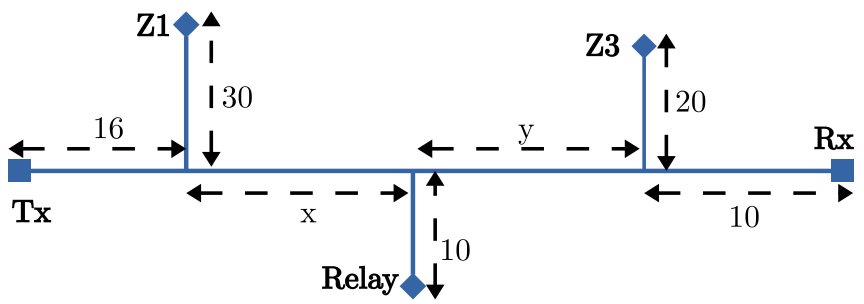

Fig. 3. PLC indoor network topology used in the simulations. The link distance is selected by enlarging and shortening $x$ and $y$, but the relay always remains at the center position.

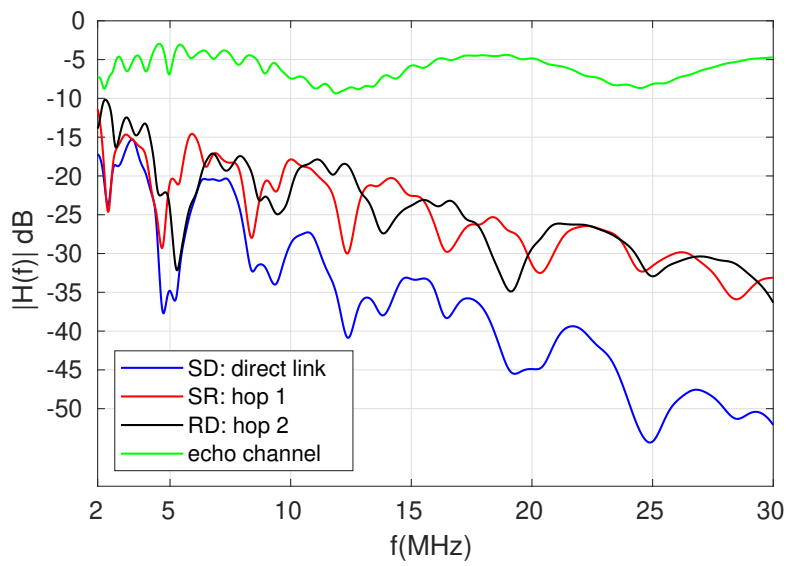

Fig. 4. Example of channel frequency responses for the topology with the shortest distance between sender and destination nodes being $200 \mathrm{~m}$.

We use the IBFD hardware design suggested in [11] and accordingly set the input impedance of the relay node to $100 \Omega$. Further, we have randomly set the loads at $\mathrm{Z} 1$ and $\mathrm{Z} 3$, which are modeled as R-L-C resonators with frequency selective impedance functions [21]. The load parameters have resulted in a resistance value of $R=853 \Omega$, a quality factor of $Q=6$, and a resonant frequency $f_{0}=2.7 \mathrm{MHz}$ for $\mathrm{Z1}$, and $R=1312 \Omega, Q=9$, and $f_{0}=10.07 \mathrm{MHz}$ for Z2. We only vary the two link lengths $x$ and $y$, shown in Fig. 3, to generate various channel frequency responses of different transmission distance (i.e. relay remains at the center and load impedance functions are fixed).

We compute the echo channel as [11]

$$
H_{\mathrm{e}}(f)=p \cdot \frac{Z_{\mathrm{PLC}}(f)-Z_{\mathrm{HY}}}{Z_{\mathrm{PLC}}(f)+Z_{\mathrm{HY}}},
$$

where $Z_{\mathrm{HY}}$ is the input impedance of the hybrid circuit at the PLC network port that is set to $100 \Omega$ as per the design in [11], $Z_{\mathrm{PLC}}(f)$ is the access impedance seen by the network node, which is computed using the PLC channel simulator for the selected scenario, and $p$ is a scaling factor that is dependent on the hybrid design, which we set as $p=0.9$ [11].

We show an example of $H_{\mathrm{sd}}, H_{\mathrm{sr}}, H_{\mathrm{rd}}$, and $H_{\mathrm{e}}$ for a $200 \mathrm{~m}$ net link length between $\mathrm{Tx}$ and $\mathrm{Rx}$ in Fig. 4. The mean gains of the generated channel responses are $\left\{H_{\mathrm{sd}}, H_{\mathrm{sr}}, H_{\mathrm{rd}}\right\}=$ $\{-37.03,-25.25,-24.98\}$ (in $\mathrm{dB}$ ) for the $200 \mathrm{~m}$ distance net- work, and $\{-56.10,-34.77,-34.53\}$ for the $400 \mathrm{~m}$ distance network. The latter values are consistent with the reported attenuation for measured channels in worst case scenarios of the OPERA project [22].

2) Noise Model: We use the noise model specified in the IEEE 1901 BB-PLC standard for the colored background noise, whose PSD reduces with frequency as $S_{\mathrm{n}}(f)=a+$ $b|f|^{c}$ [20], in $\mathrm{dBm} / \mathrm{Hz}$. We simulate two different noise conditions by varying $\{a, b, c\}=\{-145,53.23,-0.337\}$ and $\{a, b, c\}=\{-140,38.75,-0.72\}$ for high and low noise conditions, which have a floor of -100 and $-130 \mathrm{dBm} / \mathrm{Hz}$.

3) Transceiver Parameters: We use a flat transmit PSD at the source with $S_{\mathrm{s}}(f)=-55 \mathrm{dBm} / \mathrm{Hz}$ set as per the IEEE 1901 standard [20], and use the frequency range of $2-28 \mathrm{MHz}$ for communications. The set of QAM constellations size is $M=\{2,4,16,32,64,256,1024\}$. We also account for the loss in spectral efficiency due to the ratio of useful sub-carriers to all available sub-carriers, which is $917 / 1536$, and due to the inclusion of a maximum length cyclic prefix of $7.56 \mu \mathrm{s}$ in an OFDM symbol period of $48.52 \mu \mathrm{s}$.

\section{B. Results}

We present the results of the achievable data rate in Fig. 5 and Fig. 6 for the low- and high-noise conditions, respectively. Based on these results, we make the following observations.

Observation - 1 . The data rate gain provided by relayaided schemes w.r.t. the direct link are higher when the communication conditions are noisier, i.e., for lower SINR and data rates. Along the same lines, the results also support the intuition that relay-aided links are beneficial only for large transmission distances. Else, they are either unnecessary as the direct link provides sufficient performance, or are impacted by a strong crosstalk (XT) from the direct link.

Observation - 2. In the case of IBFD-DF, the overall performance is mostly limited by XT at the destination that degrades the SINR of the second hop, and therefore reduces the minimum data rate of the two links that determine the system capacity for a fixed-rate link. Due to this reason, the three IBFD-DF schemes perform similarly for link lengths below about $500 \mathrm{~m}$. In the high noise scenario, the reduction of the XT at higher distances does not result in any gain because SINR is too low. It has been tested that with a weaker direct-link, notable differences between the three IBFD-DF approaches appear.

Observation - 3. We observe that IBFD-DF outperforms IBFD-AF for the case of FD1-AF, which is the practically achievable AF implementation. The results for FD2-AF, on the other hand, may be considered as an upper bound of the achievable data rate under ideal conditions.

Observation - 4. Finally, we notice that using IBFD does not double the capacity achieved by HD even though simultaneous transmission and reception has the potential to double the bidirectional data rate. This is due to the XT interfering at the destination in FD mode. Therefore, for DF relaying, gains in data rate are observed as we approach longer transmission distances as the impact of XT reduces. 


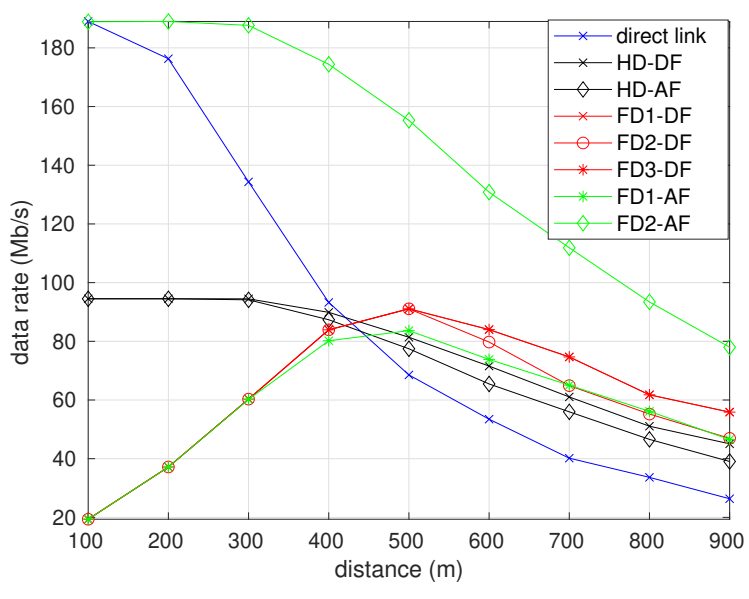

Fig. 5. Comparison of achievable data rate with different relaying techniques for the low noise condition.

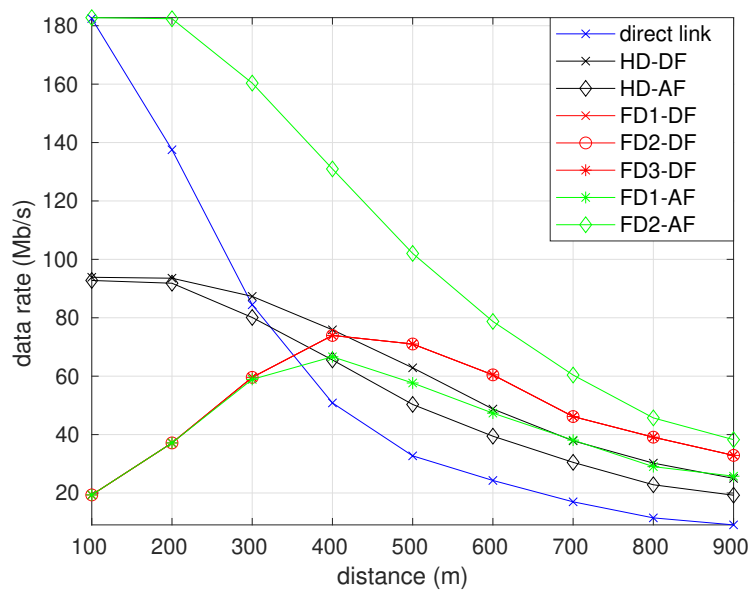

Fig. 6. Comparison of achievable data rate with different relaying techniques for the high noise condition.

\section{CONCLUSION}

In this paper, we presented the foundational analyses on the achievable performance of IBFD relay-aided PLC networks using different hopping techniques. This study guides the design strategies for future full-duplex relay networks in terms of choosing the relaying schemes to be used and the echo cancellation procedure to be implemented at the IBFD relay node based on the cost constraints, operating conditions, and desired performance. Our analyses also quantify the data rate gain and/or coverage enhancement achievable under different PLC channel and noise conditions, which in turn also suggests guidelines for placing repeaters in an in-home or a smart grid distribution network scenario. Since the study has been conducted over a simple power-network topology with few loads, channel responses are rather benign and crosstalk from the direct-link signal on the relayed one has a major impact on IBFD schemes performance. Hence, tests over more complicated PLC network models, or with measured channels, can provide more insight into this problem. We identify challenges for the practical implementation of IBFD relaying like handling the extended channel impulse response resulting from a composite S-R-D link from triple convolutions, and designing cost-efficient relays for IBFD-AF scheme that can achieve performance comparable to that of IBFD-DF methods.

\section{REFERENCES}

[1] L. Lampe, A. M. Tonello, and T. G. Swart, Power Line Communications: Principles, Standards and Applications from multimedia to smart grid. John Wiley \& Sons, 2016.

[2] S. Galli, A. Scaglione, and Z. Wang, "For the grid and through the grid: The role of power line communications in the smart grid," Proc. of the IEEE, vol. 99, no. 6, pp. 998-1027, 2011.

[3] "IEEE standard for medium frequency (less than $12 \mathrm{MHz}$ ) power line communications for smart grid applications," IEEE Std 1901.1-2018, pp. 1-192, May 2018.

[4] "Technology for powerline communication," devolo Smart Grid, https://www.devolo.com/smart-grid/plc-technology.

[5] "Our BPL communications products," Power Plus Communications, https://www.ppc-ag.com/products-and-services/bpl-products/.

[6] S. Galli, H. Latchman, V. Oksman, G. Prasad, and L. Yonge, "Multimedia PLC systems," in Power Line Communications, pp. 473-508, Wiley Online Library, 2016.

[7] L. Lampe, R. Schober, and S. Yiu, "Distributed space-time coding for multihop transmission in power line communication networks," IEEE $J$. Sel. Areas Commun., vol. 24, no. 7, pp. 1389-1400, 2006.

[8] S. D'Alessandro and A. Tonello, "Relaying protocols for in-home PLC," in MIMO Power Line Communications (L. Berger et al., eds.), ch. 20, pp. 553-572, CRC Press, 2017.

[9] L. Lampe and A. H. Vinck, "Cooperative multihop power line communications," in Proc. IEEE Int'l. Symp. Power Line Commun. Applicat. (ISPLC), pp. 1-6, 2012.

[10] X. Cheng, R. Cao, and L. Yang, "Relay-aided amplify-and-forward powerline communications," IEEE Trans. Smart Grid, vol. 4, no. 1, pp. 265-272, 2013.

[11] G. Prasad, L. Lampe, and S. Shekhar, "In-band full duplex broadband power line communications," IEEE Trans. Commun., vol. 64, no. 9, pp. 3915-3931, 2016.

[12] G. Prasad, L. Lampe, and S. Shekhar, "Digitally controlled analog cancellation for full duplex broadband power line communications," IEEE Trans. Commun., vol. 65, no. 10, pp. 4419-4432, 2017.

[13] G. Prasad and L. Lampe, "Full-duplex power line communications: Design and applications from multimedia to smart grid," IEEE Commun. Mag., pp. 2-8, 2019.

[14] T. Riihonen, S. Werner, and R. Wichman, "Optimized gain control for single-frequency relaying with loop interference," IEEE Trans. Wireless Commun., vol. 8, no. 6, pp. 2801-2806, 2009.

[15] T. Riihonen, S. Werner, and R. Wichman, "Hybrid full-duplex/halfduplex relaying with transmit power adaptation," IEEE Trans. Wireless Commun., vol. 10, no. 9, pp. 3074-3085, 2011.

[16] Y. Y. Kang, B.-J. Kwak, and J. H. Cho, "An optimal full-duplex AF relay for joint analog and digital domain self-interference cancellation," IEEE Tran. Commun., vol. 62, no. 8, pp. 2758-2772, 2014.

[17] S. Li, K. Yang, M. Zhou, J. Wu, L. Song, Y. Li, and H. Li, "Full-duplex amplify-and-forward relaying: Power and location optimization," IEEE Trans. Veh. Techn., vol. 66, no. 9, pp. 8458-8468, 2017.

[18] F. Passerini and A. M. Tonello, "Analog full-duplex amplify-and-forward relay for power line communication networks," IEEE Commun. Lett., vol. 23, no. 4, pp. 676-679, 2019.

[19] C. Wenzel, "Low frequency circulator/isolator uses no ferrite or magnet," RF Design, 1991.

[20] "IEEE standard for broadband over power line networks: Medium access control and physical layer specifications," IEEE Std 1901-2010, pp. 11586, Dec 2010.

[21] F. J. Canete, J. A. Cortes, L. Diez, and J. T. Entrambasaguas, "A channel model proposal for indoor power line communications," IEEE Commun. Mag., vol. 49, no. 12, pp. 166-174, 2011.

[22] M. Tlich, A. Zeddam, F. Moulin, and F. Gauthier, "Indoor power-line communications channel characterization up to $100 \mathrm{MHzPart}$ I: Oneparameter deterministic model," IEEE Trans. Power Del., vol. 23, no. 3, pp. 1392-1401, 2008. 